\title{
The Effect of Positive Postdebridement Cultures on Local Muscle Flap Reconstruction of the Lower Extremity
}

\author{
Arjun Kanuri, MD, MBA \\ Neil D. O'Kelly, MD \\ John Shuck, MD \\ Paul Kim, DPM \\ Karen K. Evans, MD \\ Christopher E. Attinger, MD
}

Background: Local muscle flaps are a reconstructive option for wound coverage in the distal lower extremity, particularly in high-risk patients who are poor candidates for free tissue transfer. At our institution, chronic and infected wounds are managed with serial debridement before definitive reconstruction. There is a paucity of data on optimal timing for reconstruction in this patient population. This study investigates the relationship of positive postdebridement cultures (PDC) and wound closure rates at 90 days.

Methods: A retrospective review of patients undergoing local muscle flap coverage of chronic distal lower extremity wounds between 2006 and 2012 was performed. All patients were managed with serial debridement until negative PDC were obtained. In some cases, PDC remained positive or exhibit delayed culture growth in the day(s) following closure. Data recorded include demographics, flap type/ location, culture data, and wound closure at 90-day follow-up.

Results: Of 76 patients, 60 met inclusion criteria with minimum 90-day follow-up. Despite $100 \%$ flap survival, 17 patients $(28.3 \%$ ) had failure of wound closure at 90 days and 22 patients $(36 \%)$ had positive PDC. Multivariate analysis identified positive PDC (odds ratio, 29.6; 95\% CI, 3.6-246.4; $P=0.002$ ) and smoking (odds ratio, $8.9 ; 95 \% \mathrm{CI}, 1.4-57.6 ; P=0.02$ ) as independent predictors of nonclosure at 90 days. Conclusions: In this series of local muscle flap coverage of distal lower extremity wounds, positive PDC were a strong independent predictor of failure of wound closure at 90 days. This study demonstrates the importance of serial debridement to negative cultures before definitive coverage in this patient population. (Plast Reconstr Surg Glob Open 2018;6:e1864; doi: 10.1097/GOX.0000000000001864; Published online 5 September 2018.)

\section{BACIKGROUND}

Chronic lower extremity and diabetic foot ulcers remain a reconstructive challenge, with recurrence rates after surgical management reported to be as high as $80 \%$. $^{1}$ This patient population often suffers from a wide range of comorbidities including diabetes, neuropathy, peripheral arterial disease (PAD), coronary artery disease, and kidney disease, which often makes them high-risk candidates for free tissue transfer and limits treatment options. ${ }^{2-4}$ Additionally, the cost of managing these chronic wounds is enormous and only growing. ${ }^{5}$

From the Department of Plastic Surgery, Medstar Georgetown University Hospital, Washington, D.C.

Received for publication May 3, 2018; accepted May 22, 2018.

Presented at Plastic Surgery the Meeting (ASPS) 2015 in Boston.

Copyright @ 2018 The Authors. Published by Wolters Kluwer Health, Inc. on behalf of The American Society of Plastic Surgeons. This is an open-access article distributed under the terms of the Creative Commons Attribution-Non Commercial-No Derivatives License 4.0 (CCBY-NC-ND), where it is permissible to download and share the work provided it is properly cited. The work cannot be changed in any way or used commercially without permission from the journal. DOI: 10.1097/GOX.0000000000001864
The management of these complex wounds remains controversial as some surgeons advocate for early amputation over limb salvage. ${ }^{6}$ Indeed, the variability of a surgeon's approach to diabetic limb salvage versus amputation in the United States is 8.6-fold, and up to 23\% of this population that presents to emergency rooms with a foot ulcer is managed with immediate amputation. ${ }^{7,8} \mathrm{Ag}$ gressive functional limb salvage and reconstruction have been the mainstay of treatment at our institution, given the historical data that once a major amputation has been performed, the chance of a second, contralateral amputation approaches $50 \%$ at 2 years and overall mortality approaches $80 \%$ at 5 years. ${ }^{9-13}$ In contrast, diabetics who undergo successful functional limb saving reconstruction enjoy improved survival when compared with these historical controls. ${ }^{6}$ Reconstructive surgeons should strive for functional limb sparing outcomes when managing chronic lower extremity wounds.

Disclosure: The authors have no financial interest to declare in relation to the content of this article. The Article Processing Charge was paid for by the authors. 
The exposure of vital structures including bone, tendons, joints, and neurovascular bundles combined with a paucity of local surrounding soft tissues makes closing lower extremity soft-tissue defects a significant reconstructive challenge. Because of inadequate recipient beds, simple skin grafting is often ineffective, though this may be changing with advances in negative pressure wound therapy and novel collagen scaffolds. ${ }^{14-17}$ Similarly, the utility of local random flaps is constrained by their limited reach, small size, and donor-site morbidity. ${ }^{15}$

Pedicled muscle flaps were first popularized by Ger in the 1960s as a solution for advanced stasis ulcers, posttraumatic wounds, and diabetic ulcers in the lower extremities. ${ }^{18-20}$ In the subsequent decades, the benefits of these flaps became clear and they were quickly adopted by the plastic surgery community as an excellent option for these long-standing problems. ${ }^{21,22}$ More recently, with the evolution of microsurgery and improvements in microsurgical success rates, free tissue transfer has supplanted local muscle flaps as the gold standard reconstructive option for complex distal lower extremity reconstruction. ${ }^{23}$ However, free tissue transfer requires extensive preoperative clearance, long operative times, extended hospital stays, and adequate recipient vessels. Because of these characteristics, free tissue transfer is often considered high risk and is not offered to patients with multiple major comorbidities. ${ }^{4}$ Indeed, free flap reconstruction is relatively contraindicated in diabetics with end-stage renal disease. ${ }^{4}$ Pedicled muscle flaps remain the best and only option for bringing healthy, vascularized tissue to reconstruct complex lower extremity defects in patients that are not candidates for free tissue transfer.

In this highest risk population, failure of pedicled muscle flaps to cover lower extremity wounds can lead to partial or complete amputation resulting in significant physical, emotional, and financial costs. With no other options available, failure can lead to amputation, and by corollary, increased mortality. ${ }^{24,25}$ Consequently, every effort should be made to identify correctable risk factors for failure. The presence of concomitant peripheral vascular disease and significant pedal vessel atherosclerosis makes these intrinsic muscle flaps more complex in this patient population. To our knowledge, no study has formally and systematically evaluated risk factors for failure of wound closure after local muscle flap coverage in the lower extremity.

A potential target for improvement in outcomes is that of debridement and wound bed preparation. Bacteria and biofilm have long been known to complicate reconstructive efforts, and it is currently accepted that aggressive debridement and wound bed preparation is the first step in wound management. ${ }^{26,27}$ Despite the critical role of debridement for reconstructive outcomes, no objective end point for debridement has been identified, and adequacy of debridement is largely based on the individual surgeon's subjective evaluation of the wound.

At Georgetown University Center for Wound Healing, as our limb salvage protocols have evolved over time, we have increasingly paid attention to adequate debridement including biofilm to optimize outcomes. This step in wound reconstruction cannot be overstated and likely becomes more critical with each added comorbidity. Poor host defenses, high glucose levels, and periwound blood supply likely magnify the effect of residual bacterial colonization on the ultimate reconstructive outcome. At our institution, postdebridement tissue cultures are collected at the conclusion of every debridement to assess the elimination of bacteria and biofilm from the wound bed.

This study was designed to investigate possible risk factors for failure of wound closure after local muscle flap coverage in the lower extremity and, in particular, to examine the relationship of positive postdebridement cultures (PDC) on wound closure rates. We postulate that in patients undergoing local muscle flap reconstruction of lower extremity wounds, those who have achieved negative PDC before coverage would have a higher rate of wound closure at 90 days compared with those patients whose cultures remained positive at the time of flap coverage.

\section{PATIENTS AND METHODS}

This study was approved by the Georgetown University Medical Center Institutional Review Board. Through the Georgetown Limb Salvage Registry, all patients who underwent local muscle flap reconstruction of a lower extremity soft-tissue defect between 2006 and 2012 were identified. All defects, after debridement, had exposed bone, tendon, and/or joint. Only patients with minimum 90-day follow-up were included in the analysis.

All local muscle flap reconstructions were performed by the senior authors (CEA. and KKE.). All patients underwent vascular workup including pulse examination, arterial Doppler evaluation, and/or angiography. If these tests confirmed impaired blood flow and appropriate indications were present, our vascular surgery colleagues performed revascularization using the angiosome principles whenever possible. Per our limb salvage protocols, all patients were considered for free flap reconstruction and local muscle flap reconstruction. These patients were not candidates for free flap reconstruction because of multiple major comorbidities and a high risk for adverse events with general anesthesia.

Each wound underwent an initial debridement in which semiquantitative, deep culture swabs were taken, and bone, if indicated, was sent for pathologic evaluation. The PDC were taken from the areas of heaviest colonization before debridement and also the areas that are most challenging to debride such as crevices or probing holes. These PDC identify wounds that are still microscopically colonized even when the wound clinically appears to be clean and help determine whether the wound is ready for final closure or requires further debridement. After the initial debridement, broad spectrum antibiotics were initiated and then adjusted for more specific coverage as culture results became available. Final antibiotic course was determined by Infectious Disease consultation.

All wounds were managed with serial debridement, as frequently as every 24-96 hours. During each debridement, all nonviable and infected tissue was resected and the wound copiously irrigated with at least $3 \mathrm{~L}$ of saline 
Table 1. Study Variable-related Demographics

\begin{tabular}{lc}
\hline Patients & 60 \\
Mean age & 59 \\
Mean body mass index & 32.4 \\
PAD & 22 \\
DM & 34 \\
ESRD & 6 \\
Smoking & 19 \\
\hline
\end{tabular}

through a low pulsatile irrigation system. Two surgical setups (including instruments, gloves, gowns, and drapes) were used so that the initial debrided tissue and instruments would not contaminate the postirrigation field. In every case, semiquantitative fluid or tissue cultures of the wound were taken before debridement and after debridement. Our goals of debridement were to achieve both clinically clean wounds with only viable tissue remaining and negative PDC, at which time local muscle flap reconstruction took place. In some cases, PDC remained persistently positive at the time of local muscle flap closure or exhibited growth soon after closure, and these patients comprised our study group.

The primary outcome of interest was successful wound closure at 90 days. Patients were classified as having failed closure if they still had a residual open wound at 90 days postoperatively. Given the propensity for the development of new wounds secondary to biomechanical factors and/ or progression of contributing comorbid conditions, the 90-day end point was felt to most accurately reflect a successful outcome for complete flap closure. For all patients, a chart review was conducted to assess possible risk factors for wound nonclosure after local muscle flap reconstruction including positive PDC, smoking status, infection with multidrug resistant (MDR) organisms, multiorganism infections, presence of diabetes mellitus (DM), presence of PAD, and total number of debridements before closure. Univariate and multivariate logistical regression was performed to assess the effect of these potential risk factors on wound closure at 90 days.

\section{RESULTS}

Between 2006 and 2012, a total of 60 patients were identified via the Georgetown Limb Salvage registry that underwent local muscle flap reconstruction of a lower extremity defect with a minimum 90-day follow-up. The mean age was 59 years, and the mean body mass index was 32.4. Greater than $60 \%$ of patients carried formal diagnoses of comorbid obesity, diabetes, and PAD, and $10 \%$ of patients were end stage renal disease (ESRD) (Table 1). All wounds after initial debridement had exposed bone or joint at their base.

Before reconstruction, all patients were managed with sharp serial debridement with the goal of achieving clinically clean wounds and negative PDC. An average of 3.33 debridements including debridement at the time of closure was performed per patient (range, 2-7).

Sixty pedicled muscle flap reconstructions were performed, which included 16 gastrocnemius flaps, 14 abductor digiti minimi flaps, 14 abductor hallucis brevis flaps, 10 flexor digitorum brevis flaps, 3 peroneus longus flaps, 2 extensor digitorum brevis flaps, and 1 extensor hallucis longus flap (Table 2). In addition, 24 split-thickness skin grafts were used in conjunction with these muscle flaps to complete the closures.

There were 17 complications associated with the 60 reconstructive procedures for an overall complication rate of $28.3 \%$. These included infection or skin graft loss (partial, or complete, $n=14)$, dehiscence $(n=2)$, and hematoma $(n=1)$. There were no flap losses.

At the time of reconstruction, 22 patients, or $36 \%$, had positive PDC or PDC that turned positive soon after reconstruction. At 90 days postoperatively, 43 patients, or $72 \%$, had wounds that remained closed and were healed. Despite no observed flap loss or major partial flap loss, 17 patients, or $28 \%$, had failure of wound closure indicating delayed wound healing and ongoing treatment for an open wound at 90 days postoperatively.

Potential risk factors for failure of complete wound closure at 90 days after local muscle flap reconstruction were identified a priori at the time of study design and included positive PDC, DM, PAD, smoking status, MDR bacteria, multiorganism infection, and $>2$ debridements. Univariate and multivariate logistical regression was performed to assess the effect of these risk factors on wound nonclosure.

On univariate analysis, patients with positive PDC were 20 times more likely to experience failure of wound closure at 90 days when compared to those with negative PDC [odds ratio (OR), 20.4; 95\% CI, 4.7-88.3; $P<$ 0.001]. Smoking (OR, 3.7; 95\% CI, 1.1-12.1; $P=0.03$ ) and MDR bacteria (OR, 3.8; 95\% CI, 1.1-13.2; $P=0.03$ ) were also significant predictors of failure at 90 days. DM, PAD, multiorganism infection, and number of debridements were not statistically significant (Table 3). On multivariate analysis, only positive PDC (OR, 29.6; 95\% CI, 3.6246.4; $P=0.002)$ and smoking (OR, 8.9; $95 \% \mathrm{CI}, 1.4-57.6$; $P=0.02)$ remained as statistically significant risk factors for failure of wound closure at 90 days (Table 4).

Table 2. Location of Defects and Specific Muscle Coverage

\begin{tabular}{|c|c|c|c|c|c|c|c|c|c|}
\hline & $\begin{array}{c}\text { Medial } \\
\text { Heel }\end{array}$ & $\begin{array}{c}\text { Lateral } \\
\text { Heel }\end{array}$ & $\begin{array}{c}\text { Plantar } \\
\text { Heel }\end{array}$ & Midfoot & Ankle & $\begin{array}{c}\text { Lower 1/3 } \\
\text { of Leg }\end{array}$ & $\begin{array}{c}\text { Middle } 1 / 3 \\
\text { of Leg }\end{array}$ & Knee & Total \\
\hline Gastrocnemius & - & - & - & - & - & - & - & 16 & 16 \\
\hline Flexor digitorum brevis & - & - & 10 & - & - & - & - & - & 10 \\
\hline Abductor digiti minimi & - & 14 & - & - & - & - & - & - & 14 \\
\hline Extensor digitorum brevis & - & - & - & - & 2 & - & - & - & 2 \\
\hline Abductor hallucis brevis & 14 & - & - & - & - & - & - & - & $1 \overline{4}$ \\
\hline Extensor hallucis longus & - & - & - & - & - & 1 & - & - & 1 \\
\hline Peroneus longus & - & - & - & - & - & - & 3 & - & 3 \\
\hline
\end{tabular}


Table 3. Univariate Analysis-Nonclosure at 90 days

\begin{tabular}{lccc}
\hline & OR & $\mathbf{9 5 \%} \mathbf{C I}$ & $\boldsymbol{P}$ \\
\hline Positive PDC & 20.4 & $4.7-88$ & $<0.001$ \\
Positive PDC (excluding & 62.5 & $11-344$ & $<0.001$ \\
$\quad$ surface contaminants) & & & \\
DM & 1.59 & $0.49-5.09$ & 0.43 \\
PAD & 1.84 & $0.58-5.79$ & 0.30 \\
Cigarette smoking & 3.71 & $1.1-12.2$ & 0.03 \\
Multiple organism infection & 1.58 & $0.40-6.31$ & 0.52 \\
MDR colonization & 3.88 & $1.14-13.22$ & 0.03 \\
$>$ 2 debridements & 1.45 & $0.46-4.61$ & 0.53 \\
\hline
\end{tabular}

Table 4. Multivariate Analysis-Nonclosure at 90 days

\begin{tabular}{lccc}
\hline & OR & $\mathbf{9 5 \%}$ CI & $\boldsymbol{P}$ \\
\hline Positive PDC & 29.6 & $3.56-246$ & 0.002 \\
DM & 0.37 & $0.05-2.71$ & 0.33 \\
Cigarette smoking & 8.99 & $1.40-57.6$ & 0.020 \\
PAD & 1.68 & $0.26-10.8$ & 0.58 \\
Multiple organism infection & 0.87 & $0.06-12.4$ & 0.92 \\
MDR colonization & 4.08 & $0.32-51.2$ & 0.28 \\
\hline
\end{tabular}

To further test the strength of our associations, subgroup analysis was performed, univariately, on the study group with surface contaminant cultures excluded and on the study group with gastrocnemius flaps excluded. When surface contaminant cultures were excluded, the association between positive PDC and wound failure at 90 days strengthened (OR, 62.5; 95\% CI, 11-344; $P<0.001$ ). When gastrocnemius flaps were excluded, the relationship between positive PDC and wound failure at 90 days also strengthened; every patient with negative PDC was closed at 90 days.

\section{DISCUSSION}

In this study, we present a series of 60 patients with complex lower extremity defects that were not candidates for free tissue transfer and subsequently underwent local muscle flap reconstruction for limb salvage. All wounds after debridement had exposed bone or joint at the base. Despite no flap loss, only $72 \%$ of patients had successful healing of their wounds at 90 days postoperatively. This decreased success rate compared with our previous published series is indicative of the changing indications for these muscle flaps, and their growing use in comorbid patients deemed too high risk for free tissue transfer. ${ }^{6}$

To our knowledge, this is the first study designed to examine the relationship of positive PDC on wound closure rates after local muscle flap reconstruction of the lower extremity. We found that positive PDC, smoking, and the presence of MDR bacteria were significant predictors of wound failure. Positive PDC had the strongest relationship, with those patients being 20 times more likely to experience failure of wound closure 90 days when compared to those with negative PDC (Table 3). Surprisingly, diabetes, $\mathrm{PAD}$, and the number of prior debridements were not statistically significant predictors of failure (Table 3). On multivariate analysis, only positive PDC and smoking remained as significant predictors of failure of wound closure, and the strengths of their associations persisted (Table 4).
It is well accepted that aggressive debridement and wound bed preparation are the first steps in managing a chronic wound. ${ }^{26,27}$ Despite the critical role of debridement in affecting reconstructive outcomes, no objective end point for debridement has been identified. There is a lack of clear consensus on best practices in debridement, particularly the frequency and timing, and adequacy of debridement is largely based on the individual surgeon and can vary from institution to institution. Our analysis revealed that the number of debridements did not have a significant impact on wound closure rate at 90 days, but the status of PDC was a significant predictor. Surprisingly, comorbidities frequently associated with impaired wound healing, such as diabetes and PAD, did not have a significant effect on wound closure rate (Table 3).

These data further emphasize the importance of wound bed preparation and debridement on ultimate reconstructive outcomes. There is no generalizable formula for debridement or ideal number of debridements. Rather, the quality of a debridement may be more important than frequency or quantity of debridements when concerning ultimate outcome. This analysis suggests that residual bacterial colonization of a wound after debridement may be the most significant modifiable risk factor for failure after reconstruction, and these residual bacteria may not be distinguishable on clinical examination. In particular, these results argue that serial debridement should be performed until negative cultures are achieved.

To further test the strength of our associations, subgroup analysis was performed on the study group with surface contaminant cultures (coagulase-negative Staphylococcus) excluded and on the study group with gastrocnemius flaps excluded. When surface contaminant cultures were excluded, the association between positive PDC and failure of wound closure at 90 days persisted and strengthened (Table 3).

We also performed an analysis with the gastrocnemius muscle flaps excluded. The gastrocnemius flap has historically been an excellent option for coverage of the knee particularly with periprosthetic infection, and this was the primary indication in our cohort. ${ }^{28-30}$ As many as 52\% of patients who undergo a gastrocnemius flap for coverage of an infected total knee arthroplasty (TKA) experience reinfection and require reoperation. Given the strong association between the gastrocnemius muscle flap, positive PDC, and wound breakdown, we decided to reanalyze our data with this subset of patients excluded. With gastrocnemius flaps excluded, the relationship between positive PDC and failure of wound closure at 90 days persisted and strengthened. Every patient with negative PDC was closed at 90 days.

The generalizability of these results must be discussed. Historically, and in other locations on the body, pedicled muscle flaps are regularly used to eradicate infection and to cover infected fields. ${ }^{31-33}$ One could argue that local muscle flaps of the lower extremity should behave in the same way in combating infection. We hypothesize that the type 2 blood supply of local muscle flaps and the impaired host defenses characteristic of this comorbid population make reconstruction of chronic lower extremity and diabetic foot ulcers more susceptible to residual bacterial 
colonization. Cierny has shown through his classification and treatment of osteomyelitis that host immune status can dictate host response to surgical treatment. ${ }^{34}$ The majority of the patients in this study would be classified as Cierny-Mader type $\mathrm{C}$, and residual bacteria in the wound bed may impact reconstructive outcome in these patients more than in a healthier population.

One weakness of this study is the lack of true standardization of the method of obtaining the wound cultures. This is compounded by both the heterogeneity of the wound bed and tissue culture swab technique. However, it is our standard practice to thoroughly swab the wound and in particular, areas of depth and contour that may harbor the highest levels of colonization. The authors feel that the relatively high number of positive PDC in the setting of numerous debridements is a testament to this practice but should be improved by using tissue biopsies to obtain cultures. In the future, molecular diagnostics such as pyrosequencing may prove more sensitive, objective, and consistent in identifying bacterially colonized wounds, but at this time, most institutions have yet to decipher the significance of the extensive data yielded by these methods. ${ }^{35}$ Additional weaknesses include the heterogeneity of the flaps and wounds in this cohort.

Chronic and diabetic lower extremity wounds are a frustrating problem, as high-recurrence rates in this patient population are often due to factors outside the surgeon's control. Indeed, Armstrong et al. ${ }^{36}$ estimated that only $27 \%$ of this population was compliant with a postoperative offloading protocol. Stakes are high when performing these reconstructions, as wound breakdown in this population often leads to amputation and by corollary increased morbidity and mortality. Every effort should be made to identify and modify correctable risk factors for wound breakdown. The process of debridement is one of the few variables that the surgeon can control. Unfortunately, in the literature, there is a lack of clear consensus on best practices in debridement, particularly the frequency and timing of debridement before formal reconstruction. This retrospective study suggests that using objective culture data as a guide, we can both improve the outcomes of our wound reconstructions and standardize debridement practices.

$$
\begin{array}{r}
\text { Christopher E. Attinger, } \text { MD } \\
\text { Department of Plastic Surgery } \\
\text { Medstar Georgetown University Hospital } \\
3800 \text { Reservoir Rd, 1-Bles } \\
\text { Washington, DC 20007 } \\
\text { E-mail: cattinger@aol.com }
\end{array}
$$

\section{ACIKNOWLEDGMENTS}

We would like to acknowledge Ersilia Anghel and Anagha Kumar for their assistance in preparing this article.

\section{REFERENCES}

1. Mueller MJ, Sinacore DR, Hastings MK, et al. Effect of Achilles tendon lengthening on neuropathic plantar ulcers. A randomized clinical trial. J Bone Joint Surg Am. 2003;85-A:1436-1445.

2. Attinger CE, Ducic I, Neville RF, et al. The relative roles of aggressive wound care versus revascularization in salvage of the threatened lower extremity in the renal failure diabetic patient. Plast Reconstr Surg. 2002;109:1281-1290; discussion 1291.

3. Centers for Disease Control and Prevention (CDC), National Center for Health Statistics, Division of Health Care Statistics, data from the National Hospital Discharge Survey and Division of Health Interview Statistics. http://www.cdc.gov/diabetes/statistics/hosplea/diabetes_complications/fig1_number.htm.

4. Moran SL, Salgado CJ, Serletti JM. Free tissue transfer in patients with renal disease. Plast Reconstr Surg. 2004;113:2006-2011.

5. Rice JB, Desai U, Cummings AK, et al. Burden of diabetic foot ulcers for medicare and private insurers. Diabetes Care. 2014;37: 651-658.

6. Ducic I, Attinger CE. Foot and ankle reconstruction: pedicled muscle flaps versus free flaps and the role of diabetes. Plast Reconstr Surg. 2011;128:173-180.

7. Attinger CE, Ducic I, Hess CL, et al. Outcome of skin graft versus flap surgery in the salvage of the exposed achilles tendon in diabetics versus nondiabetics. Plast Reconstr Surg. 2006;117: 2460-2467.

8. Wrobel JS, Mayfield JA, Reiber GE. Geographic variation of lower-extremity major amputation in individuals with and without diabetes in the Medicare population. Diabetes Care. 2001;24: 860-864.

9. Ikonen TS, Sund R, Venermo M, et al. Fewer major amputations among individuals with diabetes in Finland in 1997-2007: a population-based study. Diabetes Care. 2010;33:2598-2603.

10. Lavery LA, Hunt NA, Ndip A, et al. Impact of chronic kidney disease on survival after amputation in individuals with diabetes. Diabetes Care. 2010;33:2365-2369.

11. Ecker ML, Jacobs BS. Lower extremity amputation in diabetic patients. Diabetes. 1970;19:189-195.

12. Goldner MG. The fate of the second leg in the diabetic amputee. Diabetes. 1960;9:100-103.

13. Kucan JO, Robson MC. Diabetic foot infections: fate of the contralateral foot. Plast Reconstr Surg. 1986;77:439-441.

14. Patzakis MJ, Wilkins J, Moore TM. The use of antibiotics in open tibial fractures. Clin Orthop Relat Res. 1983;178:31-35.

15. Attinger C. Soft-tissue coverage for lower-extremity trauma. Orthop Clin North Am. 1995;26:295-334.

16. Molnar JA, DeFranzo AJ, Hadaegh A, et al. Acceleration of Integra incorporation in complex tissue defects with subatmospheric pressure. Plast Reconstr Surg. 2005;113:1339-1346.

17. Iorio ML, Goldstein J, Adams M, et al. Functional limb salvage in the diabetic patient: the use of a collagen bilayer matrix and risk factors for amputation. Plast Reconstr Surg. 2011;127: 260-267.

18. Ger R. The operative treatment of the advanced stasis ulcer. A preliminary communication. Am J Surg. 1966;111:659-663.

19. Ger R. The management of pre-tibial skin loss. Surgery. 1968; 63:757-763.

20. Ger R. Operative treatment of the advanced stasis ulcer using muscle transposition. A follow-up study. Am J Surg. 1970;120:376-380.

21. Vasconez LO, Bostwick J III, McCraw J. Coverage of exposed bone by muscle transposition and skin grafting. Plast Reconstr Surg. 1974;53:526-530.

22. Barfred T, Reumert T. Myoplasty for covering exposed bone or joint on the lower leg. Acta Orthop Scand. 1973;44:532-538.

23. Khouri RK, Cooley BC, Kunselman AR, et al. A prospective study of microvascular free-flap surgery and outcome. Plast Reconstr Surg. 1998;102:711-721.

24. Mayfield JA, Reiber GE, Maynard C, et al. Trends in lower limb amputation in the Veterans Health Administration, 1989-1998. J Rehabil Res Dev. 2000;37:23-30.

25. Aulivola B, Hile CN, Hamdan AD, et al. Major lower extremity amputation: outcome of a modern series. Arch Surg. 2004;139:395-399; discussion 399. 
26. Steed DL, Donohoe D, Webster MW, et al. Effect of extensive debridement and treatment on the healing of diabetic foot ulcers. Diabetic Ulcer Study Group. J Am Coll Surg. 1996;183:61-64.

27. Cornell RS, Meyr AJ, Steinberg JS, et al. Debridement of the noninfected wound. J Am Podiatr Med Assoc. 2010;100:353-359.

28. Feldman JJ, Cohen BE, May JW Jr. The medial gastrocnemius myocutaneous flap. Plast Reconstr Surg. 1978;61:531-539.

29. Greenberg B, LaRossa D, Lotke PA, et al. Salvage of jeopardized total-knee prosthesis: the role of the gastrocnemius muscle flap. Plast Reconstr Surg. 1989;83:85-9, 97.

30. Tetreault MW, Della Valle CJ, Bohl DD, et al. What factors influence the success of medial gastrocnemius flaps in the treatment of infected TKAs? Clin Orthop Relat Res. 2016;474:752-763.

31. Anthony JP, Mathes SJ, Alpert BS. The muscle flap in the treatment of chronic lower extremity osteomyelitis: results in patients over 5 years after treatment. Plast Reconstr Surg. 1991;88:311-318.
32. Meland NB, Arnold PG, Pairolero PC, et al. Muscle-flap coverage for infected peripheral vascular prostheses. Plast Reconstr Surg. 1994;93:1005-1011.

33. Dumanian GA, Ondra SL, Liu J, et al. Muscle flap salvage of spine wounds with soft tissue defects or infection. Spine (Phila Pa 1976). 2003;28:1203-1211.

34. Cierny G III. Surgical treatment of osteomyelitis. Plast Reconstr Surg. 2011;127 Suppl 1:190S-204S.

35. Rudkjøbing VB, Thomsen TR, Xu Y, et al. Comparing culture and molecular methods for the identification of microorganisms involved in necrotizing soft tissue infections. BMC Infect Dis. 2016;16:652.

36. Armstrong DG, Lavery LA, Kimbriel HR, et al. Activity patterns of patients with diabetic foot ulceration: patients with active ulceration may not adhere to a standard pressure off-loading regimen. Diabetes Care. 2003;26:2595-2597. 